\title{
Evaluation of Oxidative Stress and some Biochemical Parameters in Normal Pregnant Women
}

\author{
Musher I. Kakey ${ }^{1}$, Kamaran K. Abdoulrahman ${ }^{2}$ and Gulbahar R. Uthman ${ }^{2}$ \\ ${ }^{1}$ Department of Chemistry, Faculty of Science and Health, Koya University, \\ Koya KOY45, Kurdistan Region - F.R. Iraq \\ ${ }^{2}$ Department of Chemistry, College of Science, Salahaddin University, \\ Erbil, Kurdistan Region - F.R. Iraq
}

\begin{abstract}
Pregnancy is associated with many metabolic changes in normal pregnant woman, this leads to change in physiological, biochemical, and hematological parameters drastically. The test subjects were selected among those attending to Maternity Teaching Hospital in Erbil Governorate between March 2017 and August 2017. Four groups of individuals were included in this study, 230 pregnant women divided into three groups (Group 1 first trimester, Group 2 second trimester, and Group 3 third trimester) and Group 4 contained 90 nonpregnant women as control. Full automatic chemical analyzer (Cobas C311, Germany) was used to determine the biochemical parameters. The $E L \times 800$ Absorbance Microplate Reader from BioTek (USA) instruments is used to measure the activity of superoxide dismutase (SOD), catalase (CAT), and malondialdehyde (MDA) in serum. The results of this study showed a significant increase in serum albumin, glucose, total bilirubin, direct bilirubin, alanine aminotransferase, aspartate aminotransferase, alkaline phosphatase, creatinine, urea, cholesterol, low-density lipoprotein, and MDA in pregnant women as compared to the control group, also show significant decrease in high-density lipoprotein (HDL) level, SOD activity, and CAT activity in pregnant women as compared to nonpregnant women, and nonsignificant difference in uric acid, and HDL.
\end{abstract}

Index Terms-Pregnancy, Oxidative stress, Liver parameters, Lipid profiles, Kidney function.

\section{INTRODUCTION}

More than 200 million women become pregnant every year, and in most cases, the outcome of labor is successful. Due to the hormonal status changes during pregnancy, various adaptive mechanisms are initiated due to sex hormones. Among them, change of the energetic metabolism to lipid metabolism is observed (Merabishvili, et al., 2006). Oxidative stress occurs due to an imbalance between the reactive

ARO-The Scientific Journal of Koya University

Vol. VIII, No.1 (2020), Article ID: ARO.10541, 7 pages

DOI: 10.14500 /aro.10541

Received 24 June 2019; Accepted 06 February 2020

Regular research paper: Published 20 February 2020

Corresponding author's e-mail: musher.ismael@koyauniversity.org

Copyright (c) 2020 Musher I. Kakey, Kamaran K. Abdoulrahman and Gulbahar R. Uthman. This is an open access article distributed under the Creative Commons Attribution License. oxygen species and the antioxidant levels (Miao and Clair, 2009). Oxidants (free radical) are normal products of aerobic metabolism, including superoxide radical, hydroxyl radical, and hydrogen peroxidase. They can be harmful when they produced in excess amounts (Saikumar, et al., 2013). The liver has many important functions, including metabolism, detoxification, and formation of important compounds, including blood clotting factors and albumin (Ali, 2015). The pregnant woman experiences physiological changes to support fetal growth and development. During pregnancy, the serum estrogen and progesterone levels increase progressively and reach a maximum during the third trimester. These sex steroids have effects on metabolic, synthetic, and excretory hepatic functions (Gohel, et al., 2013). During pregnancy, an increase in the levels of estrogens and progesterone will occur. These sex hormones have effects on hepatic metabolism, synthesis, and excretory functions (González, et al., 2000). Pregnant women develop physiological dyslipidemia that can be measured in the laboratory by the lipid indexes; total cholesterol (TC), triglycerides (TG), highdensity lipoprotein (HDL), and low-density lipoprotein (LDL) (Merabishvili, et al., 2006). The alterations of serum lipid indexes are associated with the gestational age. Pregnancy is accompanied by significant variations in maternal lipid metabolism (Parchwani, and Patel, 2011). During normal pregnancy, women show an increase in lipid levels, including levels of TG and TC as gestational age progresses (Ogura, et al., 2002). Both TG and TC are taken up by the placenta, metabolized and transported to the fetus in various forms; this shows that both lipids are essential for the development of the fetus. An increase in maternal lipid profile during pregnancy differs with gestational age (Woollett, 2011). It has been observed that the concentration of serum $\mathrm{TC}$, serum TG, HDL cholesterol, and LDL cholesterol in normal pregnant women increased with increasing gestational age (Herrera, 2002). Any increase in the maternal lipid profile in the third trimester is in response to the maternal switch from carbohydrate to fat metabolism which is an alternative pathway for energy generation due to high energy demand. Creatinine and blood urea nitrogen (BUN) are waste products removed from the blood by the kidneys. Creatinine is a breakdown product of creatine phosphate in the muscle. 
Serum creatinine is a marker used for renal function assessment. When the kidney function is lost, the level of BUN will be increased, but other factors may affect the BUN level (Kamili, et al., 2013; Patel, et al., 2013).

The aim of the present study is to evaluate the oxidative stress, enzymatic antioxidant activities, and some biochemical parameters in the normal pregnant women at first, second, and third trimesters) as a comparison to nonpregnant women.

\section{Materials AND Methods}

\section{A. Samples}

The test subjects were selected among those attending to Maternity Teaching Hospital in Erbil Governorate between March 2017 and August 2017. Four groups of individuals were included in this study. Group 1 contained 85 pregnant women in first trimester of pregnancy (1-3 months). Group 2 consisted of 75 pregnant women in second trimester of pregnancy (4-6 months). Group 3 comprised of 70 pregnant women in third trimester of pregnancy (7-9 months) and Group 4 contained 90 nonpregnant women as control group in this study. The blood was drawn into a tube then centrifuged at 5000 RPM and the serum was used to estimation of oxidative stress, lipid profile, liver parameters, and kidney function test. Full automatic chemical analyzer (Cobas C311, Germany) was used to determine the biochemical parameters. Superoxide dismutase (SOD) activity was assayed using (Randox-Ransod kits, USA) (colorimetric method). Catalase (CAT) activity was assayed using (Cayman kits, USA). Serum malondialdehyde (MDA) level was estimated by manual method of thiobarbituric acid reactive substances (Chaudhary, et al., 2003).

\section{B. Statistical Analysis}

Data analysis was performed with the GraphPad Prism software. The results for continuous variables are mean+SEM, analysis of variance was used in the assessment of the significance of the difference between group means.

\section{Results AND Discussion}

This study included 230 pregnant women with age ranged from 23 to 40 years. The mean age was $33 \pm 4.5$ years with no risk factors and 90 nonpregnant women as control group. The results of the current study are showing in Tables I and II.

The body produces different enzymes, including SOD, CAT, glutathione peroxidase (GSHPX), and glutathione reductase.

Table I and Fig. 1 show a significant increase in concentrations of serum MDA in the test group in comparison with the control group $(P \leq 0.05)$. Saikumar, et al. determined a high level of $\operatorname{MDA}^{14}$ (Saikumar, et al., 2013), which is similar to our result. The increased MDA levels are known to be due to increased generation of reactive oxygen species and increased oxygen demand along with a reduction in activities of enzymes like SOD, GSHPX, and CAT.

Furthermore, the results showed a significant decrease in SOD activity and CAT activity in pregnant women as compared to the control group, as shown in Table I and Figs. 2 and 3, respectively.

The mean levels of biochemical parameters were measured in healthy pregnant women and control group. These data show that the mean level of serum glucose in pregnant women

TABLE I

Oxidative Stress Parameters in Pregnant Women and Control Group

\begin{tabular}{|c|c|c|c|c|}
\hline Parameters & Control group & 1 trimester & 2 trimester & 3 trimester \\
\hline Superoxide dismutase (IU/gHb) & $705.0 \pm 28.39$ & $545.5 \pm 38.59$ & $507.9 \pm 43.07$ & $456.8 \pm 30.68$ \\
\hline Catalase (IU/gHb) & $8.378 \pm 0.394$ & $7.031 \pm 0.254$ & $6.329 \pm 0.337$ & $5.756 \pm 0.311$ \\
\hline Malondialdehyde (nmol/ml) & $1.598 \pm 0.062$ & $1.834 \pm 0.036$ & $2.672 \pm 0.098$ & $3.457 \pm 0.114$ \\
\hline
\end{tabular}

TABLE II

Biochemical Parameters in Pregnant and Control Group

\begin{tabular}{|c|c|c|c|c|}
\hline \multirow[t]{2}{*}{ Parameters } & \multirow[t]{2}{*}{ Control } & \multicolumn{3}{|c|}{ Pregnant group } \\
\hline & & $1^{\text {st }}$ trimester & $2^{\text {nd }}$ trimester & $3^{\text {rd }}$ trimester \\
\hline Aspartate aminotransferase & $19.79 \pm 1.400$ & $20.02 \pm 0.619$ & $24.71 \pm 1.810$ & $27.09 \pm 2.117$ \\
\hline Alanine aminotransferase & $13.25 \pm 0.880$ & $20.13 \pm 2.036$ & $25.09 \pm 2.870$ & $36.07 \pm 5.188$ \\
\hline Total bilirubin & $0.528 \pm 0.021$ & $1.144 \pm 0.060$ & $1.666 \pm 0.146$ & $1.876 \pm 0.215$ \\
\hline Albumin & $4.766 \pm 0.124$ & $4.034 \pm 0.119$ & $4.005 \pm 0.275$ & $4.078 \pm 0.133$ \\
\hline Direct bilirubin & $0.132 \pm 0.008$ & $0.282 \pm 0.019$ & $0.424 \pm 0.046$ & $0.501 \pm 0.046$ \\
\hline Glucose & $107.2 \pm 4.543$ & $157.9 \pm 11.23$ & $171.7 \pm 16.17$ & $209.8 \pm 13.59$ \\
\hline Creatinine & $0.597 \pm 0.027$ & $0.687 \pm 0.036$ & $0.869 \pm 0.061$ & $0.905 \pm 0.035$ \\
\hline Urea & $23.78 \pm 2.323$ & $27.94 \pm 1.868$ & $35.97 \pm 3.056$ & $34.82 \pm 1.736$ \\
\hline Uric acid & $3.970 \pm 0.144$ & $4.251 \pm 0.197$ & $4.743 \pm 0.357$ & $4.421 \pm 0.192$ \\
\hline Total cholesterol & $146.6 \pm 3.841$ & $201.4 \pm 12.21$ & $208.1 \pm 15.46$ & $240.8 \pm 7.850$ \\
\hline Triglycerides & $93.29 \pm 3.271$ & $205.4 \pm 14.19$ & $236.1 \pm 29.86$ & $247.3 \pm 17.69$ \\
\hline
\end{tabular}


is increased continuously (Table II and Fig. 4). It is in pregnant women significantly higher compared to the control group.

The mean levels of serum TC, TG, and LDL of the test subjects in the pregnant women were higher than those of the control group as shown in Table II and Figs. 5-7, and the mean level of serum HDL in pregnant women in first and second trimester is nonsignificantly decreased as compared to the control group, and the level of HDL showed significantly decreased in the third trimester as compared to the control group, as shown in Table II and Fig. 8. The placenta produces lipid peroxides which are secreted mainly on the maternal side of the placenta and remain in the maternal circulation for some time and increased lipid peroxidation markers are observed during normal pregnancy (Erişir, et al., 2009).

Elevation in TG, TC, and LDL may be due to an increase in hepatic lipase activity and a decrease in lipoprotein lipase activity. Hepatic lipase is responsible for the increased synthesis of TG at the hepatic level, whereas the decreased activity of lipoprotein lipase is responsible for the decreased catabolism at the adipose tissue. The second step of uptake of the chylomicron remnants by the liver is delayed so it leads to accumulation of TGs in plasma (Aziz and Mahboob, 2007). Hormonal variations during pregnancy affect lipid metabolism. The endogenous female sex hormones have a significant effect on serum lipids (Brizzi, et al., 1999). Increased TG plays a part to decrease HDL cholesterol. HDL particles transport cholesterol from peripheral tissues to liver. Impaired transport of cholesterol from peripheral tissues to the target area of utilization may cause the decrease in HDL cholesterol in serum. Hypertriglyceridemia leads to low HDL cholesterol; this may be due mainly to the actions of cholesteryl ester transfer protein (Pirzado, et al., 1999). Hormonal changes during pregnancy, for example, hyperestrogenemia are well documented and contribute to hyperlipidemia (Festus, et al., 2011). Decline in HDL is associated with elevated TC and LDL levels (Welty, 2013). Cholesterol level also increases in pregnancy due to elevated steroid hormone synthesis (Parchwani and Patel, 2011). Therefore, in pregnancy serum TC and LDL cholesterol are elevated. The mean level of serum alanine aminotransferase (ALT) activity was continuously increased during pregnancy (Table II and Fig. 9). It is significantly higher in pregnant women than that of control group $(P<0.05)$. The mean level of serum aspartate aminotransferase (AST) is also increased continuously but not significantly (Table II and Fig. 10). It has been reported that elevation of ALT and AST is associated with pregnancy in different cases such as intrahepatic cholestasis of pregnancy, hyperemesis gravidarum, eclampsia, hepatic infarction, acute fatty liver of pregnancy, and acute viral hepatitis (Westbrook, et al., 2016). An increase in ALT and AST levels was also found during labor, which might be caused by contractions of uterine muscle (Loganathan, et al., 2005). The mean level of serum alkaline phosphatase (ALP) activity was increased continuously during pregnancy (Table II and Fig. 11). It is significantly higher in pregnant women compared to the control group. Total ALP activity in serum almost doubles during normal pregnancy, but much of the increase is attributable to heat-stable placental ALP isoenzymes (Cunningham, 2005).

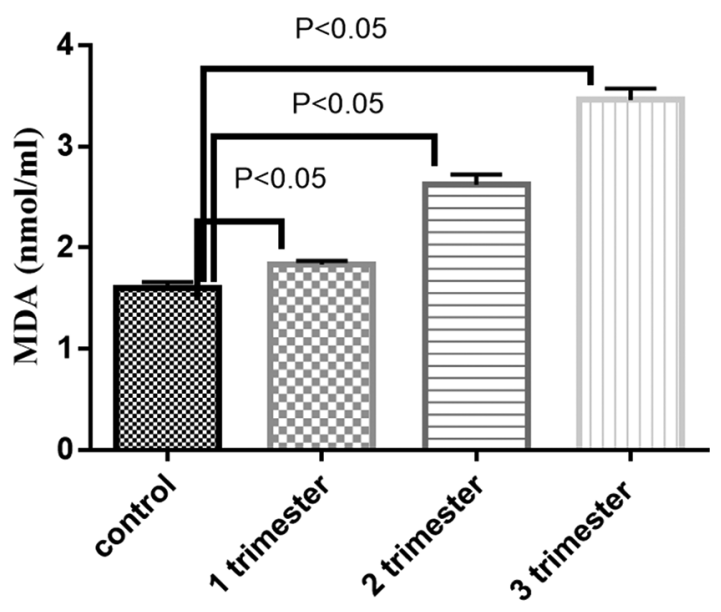

Fig. 1. Malondialdehyde level in pregnant and control group.

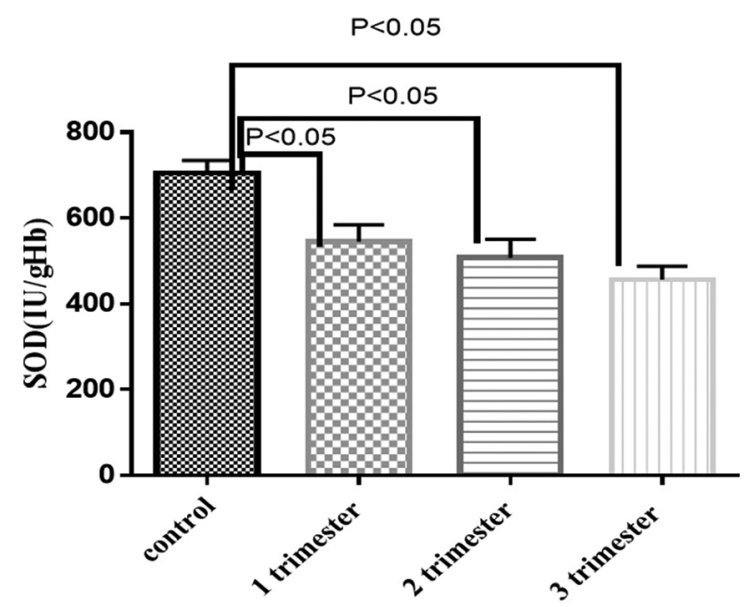

Fig. 2. Superoxide dismutase activity in pregnant and control group.

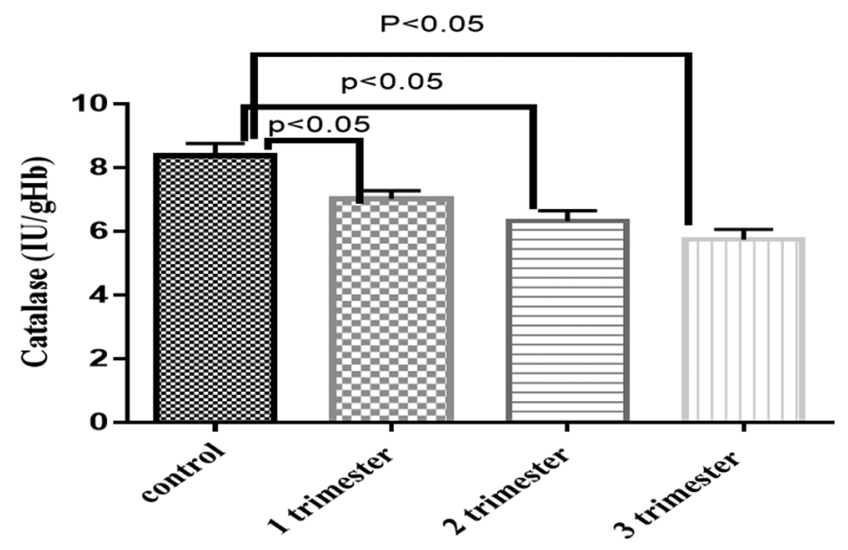

Fig. 3. Catalase activity in pregnant and control group.

Due to the lack of specificity, the measurement of serum ALP activity is a poor test for the diagnosis of cholestasis during the third trimester of pregnancy. There is no significant change in serum total protein concentration. However, serum albumin level was significantly lower in all three trimesters compared to nonpregnant women. These results are in agreement with previous works as in Table II and Figs. 12 and 13 (Festus, et al., 2011). Furthermore, these data show 


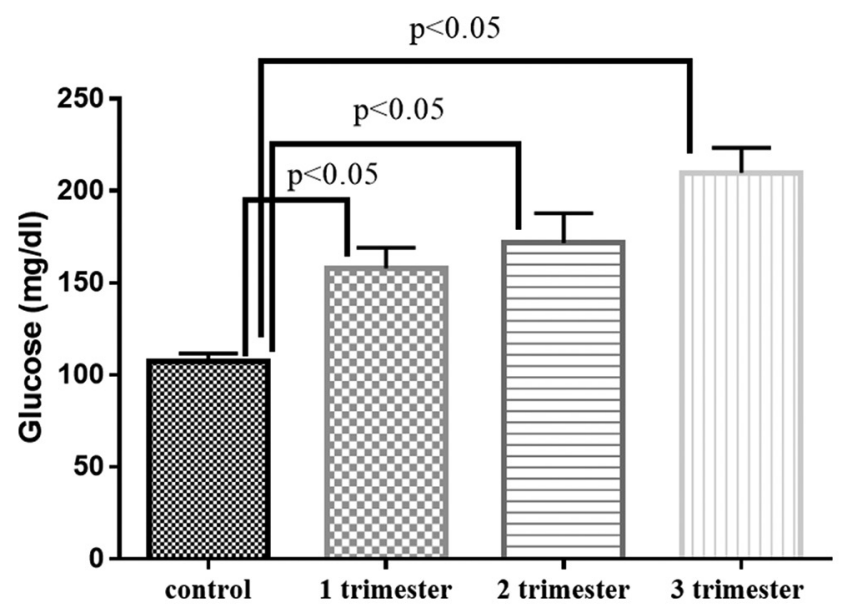

Fig. 4. Glucose level in pregnant and control group.

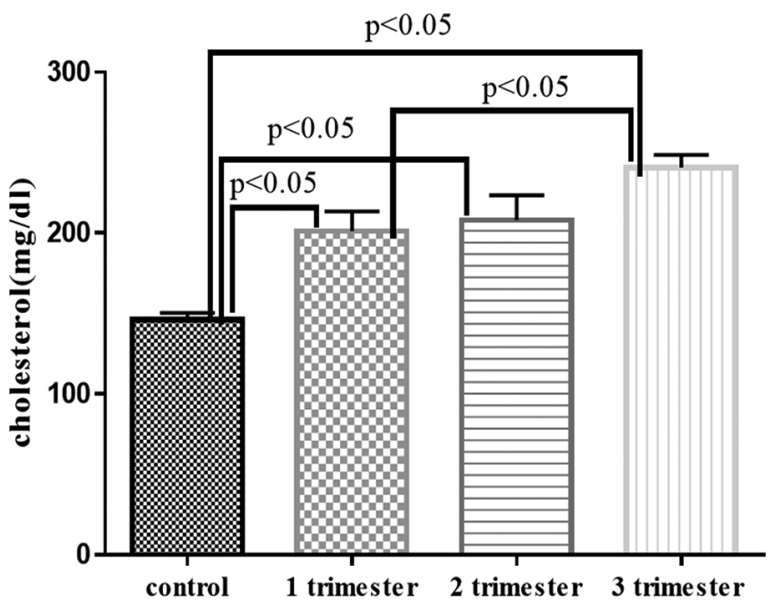

Fig. 5. Cholesterol level in pregnant and control group.

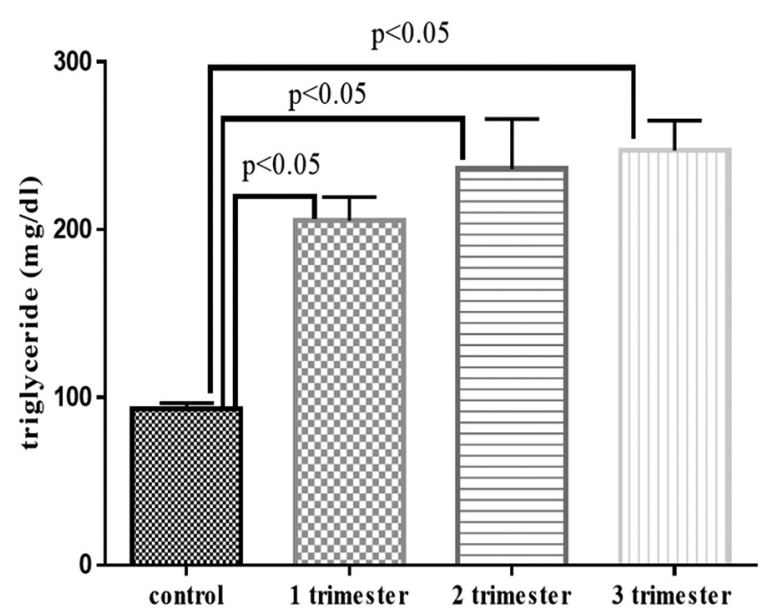

Fig. 6. Triglyceride level in pregnant and control group.

that the mean levels of total bilirubin and direct bilirubin are significantly elevated in pregnant women compared to the control group (Table II and Figs. 14 and 15). They are also increased continuously during all three trimesters.

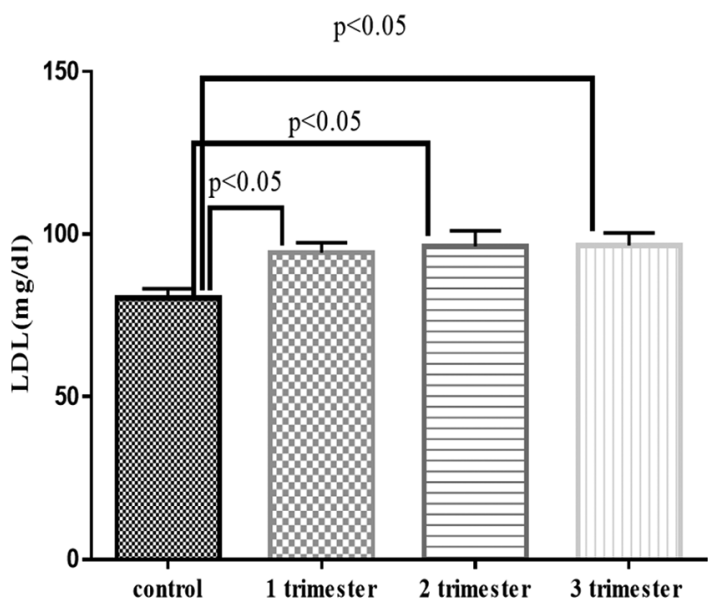

Fig. 7. Low-density lipoprotein level in pregnant and control group.

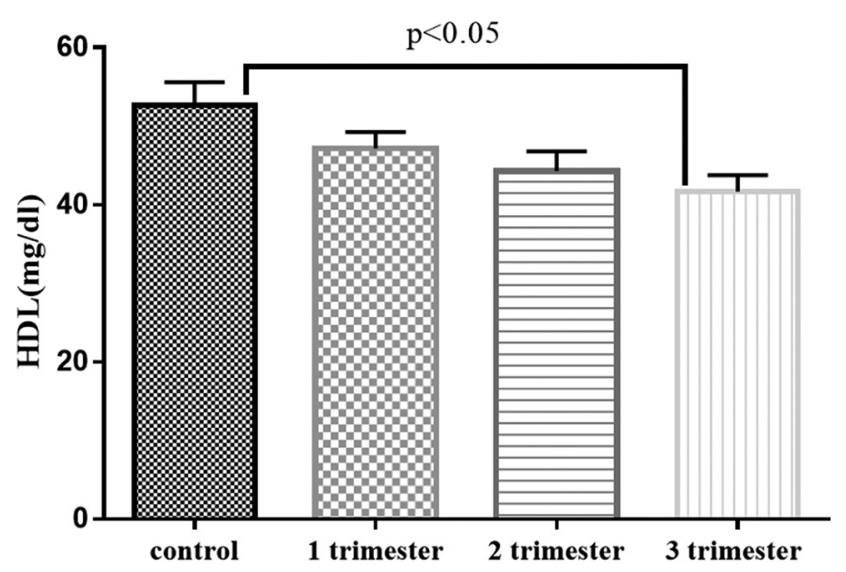

Fig. 8. High-density lipoprotein level in pregnant and control group.

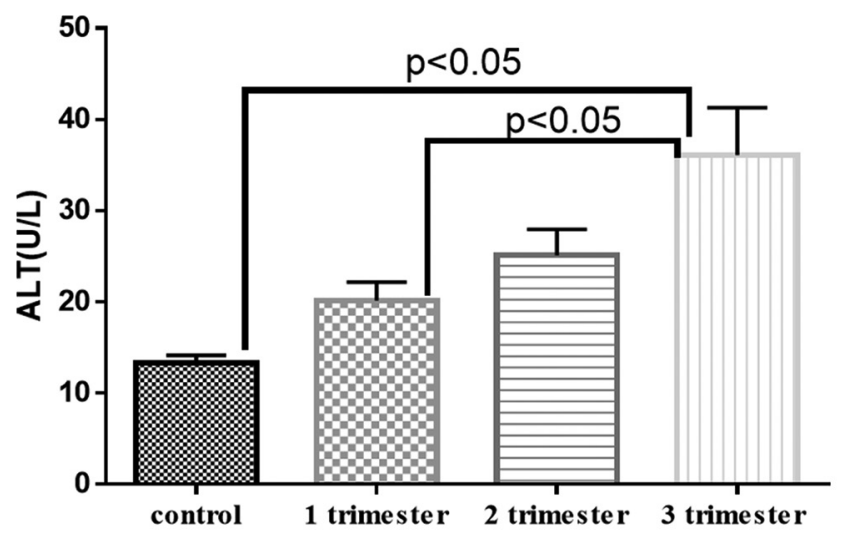

Fig. 9: Alanine aminotransferase level in pregnant and control group.

Our data are in agreement with those of Duraiswamy, et al., 2016, who reported that the level of bilirubin had increased during pregnancy in the past century; it has been reported that elevation of bilirubin in pregnant women was associated with acute viral hepatitis (Hay, 2008). Duraiswamy, et al., 2016, showed that hyperbilirubinemia is observed in 


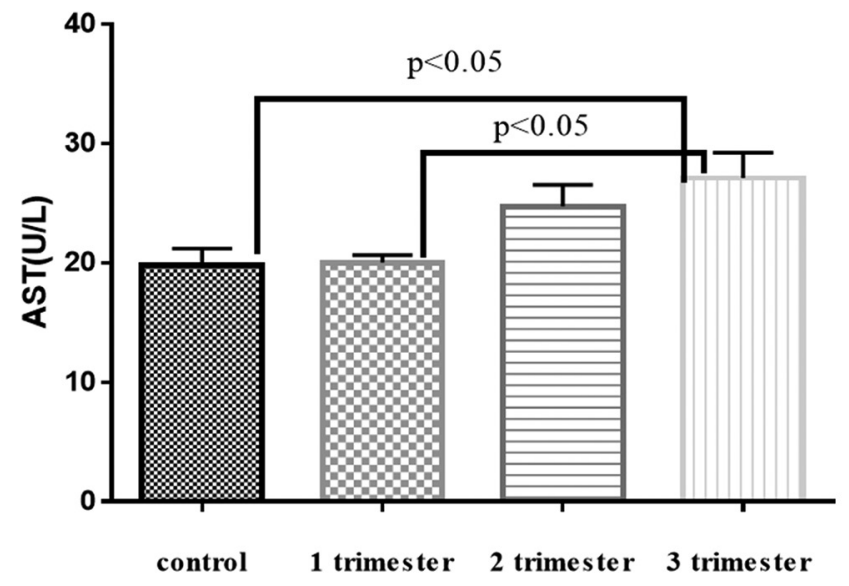

Fig. 10: Aspartate aminotransferase level in pregnant and control group.

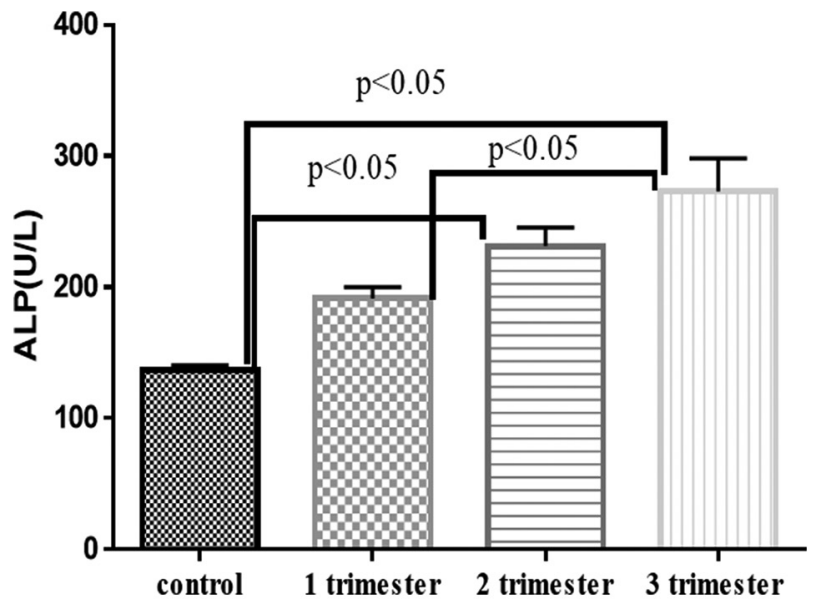

Fig. 11. Alkaline phosphatase level in pregnant and control group.

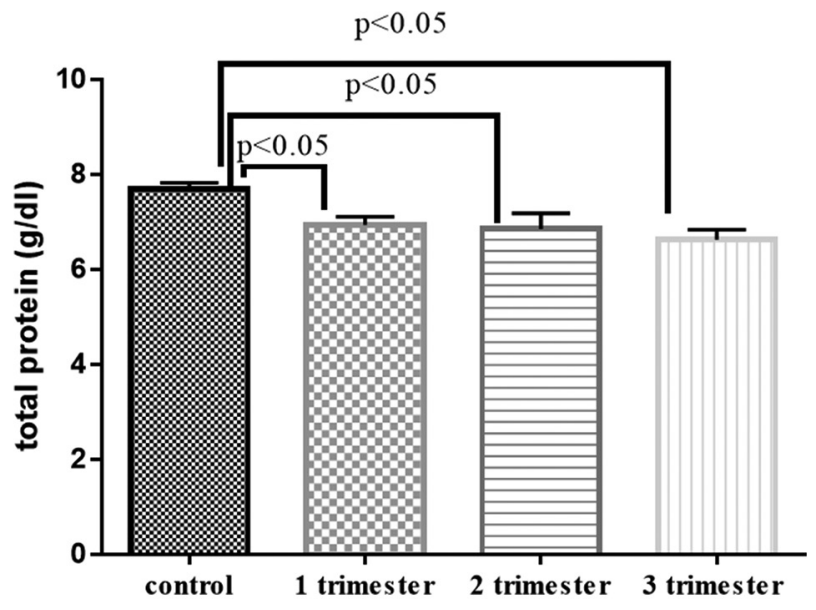

Fig. 12. Total protein level in pregnant and control group.

pregnant women who were suffering mostly from gallstonerelated disease, pre-eclampsia/eclampsia, and hyperemesis gravidarum.

This study shows that the mean levels of serum creatinine and urea are significantly increased in pregnant women as compared to the control group, whereas the mean level of

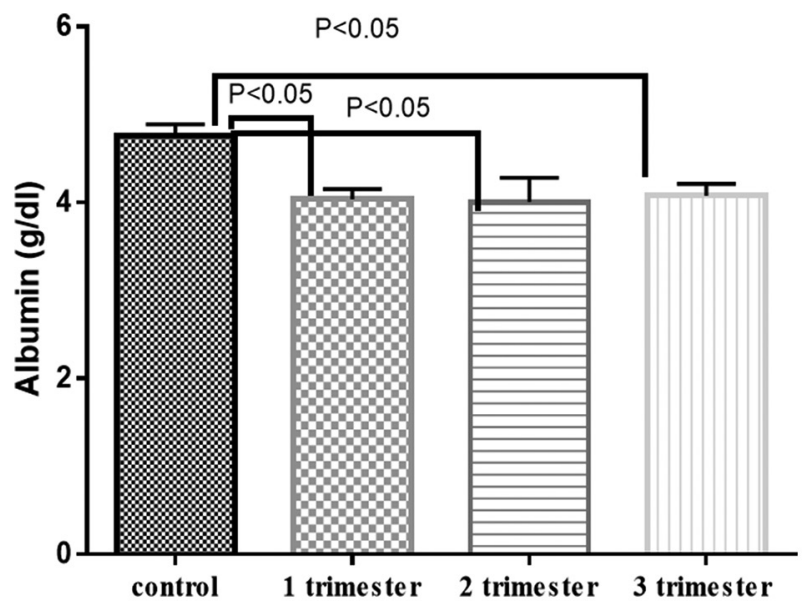

Fig. 13. Albumin level in pregnant and control group.

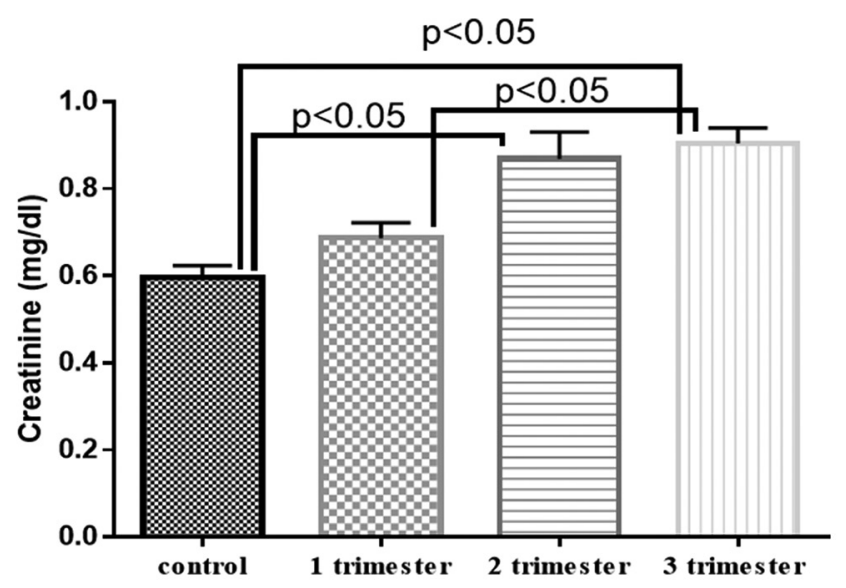

Fig. 14. Creatinine level in pregnant and control group.

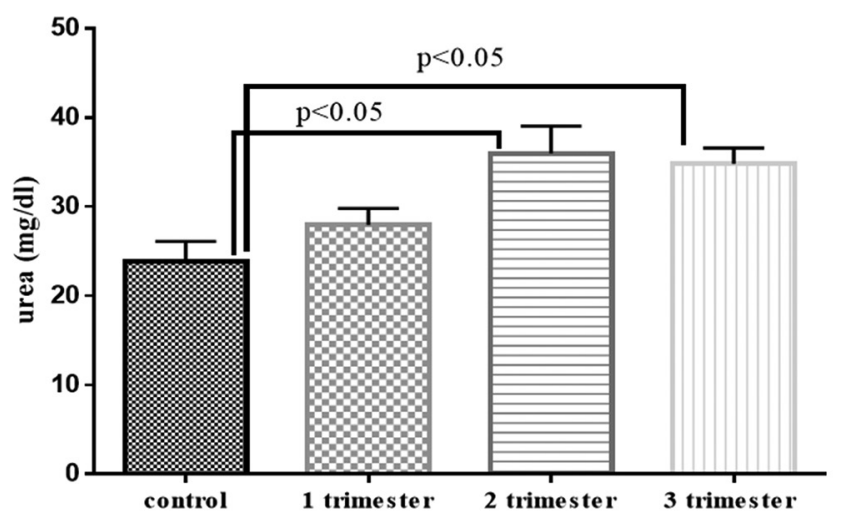

Fig. 15. Urea level in pregnant and control group.

uric acid shows nonsignificant increases in pregnant women as compared to the control group (Table II and Figs. 16-18). These data revealed that protein catabolism and high need for energy by pregnant women during the late trimester may affect the catabolism of protein in the body resulting to an increase in urea and creatinine levels. 


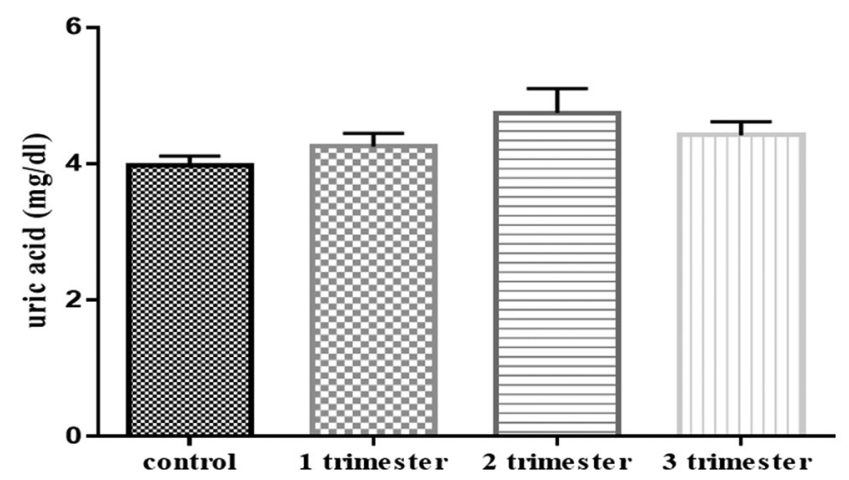

Fig. 16. Uric acid level in pregnant and control group.

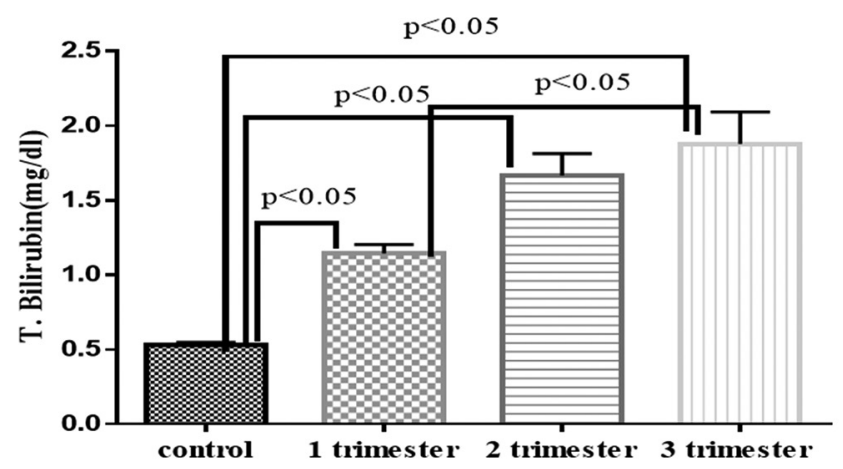

Fig. 17. T. bilirubin level in pregnant and control group.

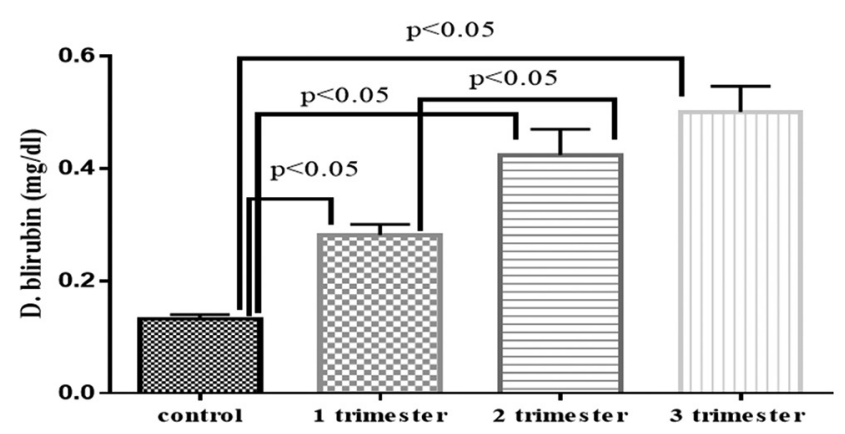

Fig. 18. D. bilirubin level in pregnant and control group.

\section{CONCLUSIONS}

As shown in this study, antioxidant enzymes activity (SOD and CAT) decrease and MDA level raised in pregnant women when compared to the control group. Glucose production is increased from the onset of pregnancy to the last trimester as compared to the control group. The results demonstrated that normal pregnancy is associated with raised TG, cholesterol, and LDL, as compared to the control group and a significant decrease in HDL in normal pregnant women as compared to the control group. Elevated levels of serum bilirubin (D. bilirubin and T. bilirubin) and hepatic enzymes ALT, AST, and ALP were seen in pregnant women cases. The results showed a significant increase in serum creatinine and urea and a nonsignificant increase in uric acid level in pregnant women as compared to the control group.

\section{ACKNOWLEDGMENTS}

The authors would like to thank the Maternity Teaching Hospital in Erbil Governorate for providing the equipment to accomplish this work and also thank all those pregnant women that participate in this work.

\section{REFERENCES}

Ali, D.M. 2015. Effect normal pregnancy and duration on liver enzymes tests. Global Journal of Medical Research, 15(1), pp.8-12.

Aziz, R., and Mahboob, T. 2007. Pre-eclampsia and lipid profile. Pakistan Journal of Medical Sciences, 23(5), pp.751.

Brizzi, P., Tonolo, G., Esposito, F., Puddu, L., Dessole, S., Maioli, M., and Milia, S. 1999. Lipoprotein metabolism during normal pregnancy. American Journal of Obstetrics and Gynecology, 181(2), pp.430-434.

Chaudhary, L., Tandon, O.P., Vaney, N., and Agarwal, N. Lipid peroxidation and antioxidant enzymes in gestational diabetics. Indian Journal of Physiology and Pharmacology, 47(4), pp.441-446.

Cunningham, F.G. 2005. Maternal physiology. Williams Obstetrics, 2005, pp.121-150.

Duraiswamy, S., Sheffield, J.S., Mcintire, D., Leveno, K., and Mayo, M.J. 2017. Updated etiology and significance of elevated bilirubin during pregnancy: changes parallel shift in demographics and vaccination status. Digestive Diseases and Sciences, 62(2), pp.517-525.

Erişir, M., Benzer, F., and Kandemir, F.M. 2009. Changes in the rate of lipid peroxidation in plasma and selected blood antioxidants before and during pregnancy in ewes. Acta Veterinaria Brno, 78(2), pp.237-242.

Festus, O.O., Idonije, O.B., Eseigbe, M.A., Okhiai, O., Unuabonah, F., and Dike, M. 2011. Comparative study of lipid profile of normal pregnant women in the different trimesters. Archives of Applied Science Research, 3(3), pp.528-532.

Gohel, M.G., Joshi, A.G., Anand, J.S., Makadia, J.S., and Kamariya, C.P. 2016. Evaluation of changes in liver function test in first, second and third trimester of normal pregnancy. International Journal of Reproduction, Contraception, Obstetrics and Gynecology, 2(4), pp.616-20.

González, I.M., Armada, E.R., Díaz, J.R., García, P.D., García, M.M., González, A.G., Fernández, C.B., Iñiguez, A.R., and Rayo, I.L. 2000. Practice guidelines of the spanish society of cardiology for the management of cardiac disease in pregnancy. Revista Espanola de Cardiologia, 53(11), pp.1474-95.

Hay, J.E. 2008. Liver disease in pregnancy. Hepatology, 47(3), pp.1067-1076.

Herrera, E. 2002. Lipid metabolism in pregnancy and its consequences in the fetus and newborn. Endocrine, 19(1), 43-55.

Kamili, A., Bengoumi, M., Oukessou, M., Faye, B., and Lefebvre, H.P. 2013. Assessment of glomerular filtration rate in normally hydrated and dehydrated dromedary camel by plasma exogenous creatinine clearance test. Emirates Journal of Food and Agriculture, 25(4), pp.314-319.

Loganathan, G., George, R., Eapen, C.E., Mathai, M., Jasper, P., Seshadri, L., Shankar, V., Paul, S., Joseph, G., Balasubramanian, K.A., and Chandy, G.M. 2005. Liver function tests in normal pregnancy: A study from Southern India. Indian Journal of Gastroenterology: Official Journal of the Indian Society of Gastroenterology, 24(6), pp.268-269.

Merabishvili, N.V., Kamladze, S.O., and Sulaberidze, G,T. 2006. Peculiarities of lipid metabolism during pregnancy. Georgian Medical News, 138, pp.86-89.

Miao, L., and Clair, D.K. 2009. Regulation of superoxide dismutase genes: Implications in disease. Free Radical Biology and Medicine, 47(4), pp.344-56.

Ogura, K., Miyatake, T., Fukui, O., Nakamura, T., Kameda, T., and Yoshino, G. 2002. Low-density lipoprotein particle diameter in normal pregnancy and preeclampsia. Journal of Atherosclerosis and Thrombosis, 9(1), pp.42-7. 
Parchwani, D., and Patel, D. 2011. Status of lipid profile in pregnancy. National Journal of Medical Research, 1(1), pp.10-12.

Patel, S.S., Molnar, M.Z., Tayek, J.A., Ix, J.H., Noori, N., Benner, D., Heymsfield, S., Kopple, J.D., Kovesdy, C.P., and Kalantar-Zadeh, K. 2013. Serum creatinine as a marker of muscle mass in chronic kidney disease: Results of a cross- sectional study and review of literature. Journal of Cachexia, Sarcopenia and Muscle, 4(1), pp.19-29.

Pirzado, Z.A., Sngi, S.A., and Malik, R. 1999. High density lipoprotein cholesterol (HDL) metabolism and its role in ischemic heart disease. Pakistan Journal of Medical Research, 38(1), pp.38-41.
Saikumar, P., Jaya, D., and Devi, M.R. 2013. Oxidative stress in pregnancy. ISOR Journal of Dental and Medical Sciences, 3, pp.12-13.

Welty, F.K. 2013. How do elevated triglycerides and low HDL-cholesterol affect inflammation and atherothrombosis? Current Cardiology Reports, 15(9), pp. 400 .

Westbrook, R.H., Dusheiko, G., and Williamson, C. 2016. Pregnancy and liver disease. Journal of Hepatology, 64(4), pp.933-945.

Woollett, L.A. 2011. Transport of maternal cholesterol to the fetal circulation. Placenta, 1(32), pp.218-221. 